\title{
Influence of a modified drawing process on the resulting residual stress state of cold drawn wire
}

\author{
Markus Baumann ${ }^{1 *}$, Alexander Graf ${ }^{1}$, René Selbmann ${ }^{2}$, Katrin Brömmelhoff $^{3}$, Verena Kräusel $^{1}$, Dirk Landgrebe $^{1}$, and \\ Markus Bergmann ${ }^{2}$ \\ ${ }^{1}$ Professorship for Forming and Joining, Chemnitz University of Technology, 09107 Chemnitz, Germany \\ ${ }^{2}$ Fraunhofer Institute for Machine Tools and Forming Technology, 09126 Chemnitz, Germany \\ ${ }^{3}$ Institute for Materials Science and Technology - Metallic Materials, Technical University Berlin, 10587 Berlin, Germany
}

\begin{abstract}
Torsion bars are used in automotive engineering as well as in other industrial applications. Such elements are produced by bending cold drawn wires. In conventional drawing processes tensile residual stresses occur near the surface of the wire. Small bending radii, which are required in limited assembly spaces, result in component failure due to reduced formability. Additional operations such as heat treatment or shot peening are necessary to influence the residual stress of the wire and to improve the dynamic stability of the torsion bar. The aim of the research is to reduce tensile residual stresses near the surface of the wire in order to eliminate process steps and to enhance formability. Therefore, a forming technology is developed by using a modified drawing die geometry on the basis of gradation extrusion. Finite element simulation is used to investigate the influences of element geometry, number of elements and process modification on the resulting residual stresses after wire drawing of a steel alloy. The results are evaluated and compared with the conventional wire drawing process. Furthermore, the requirements for the design of an experimental test device will be outlined as well as the measurement of the residual stresses by using X-ray diffraction.
\end{abstract}

Keywords. Residual stress, Cold forming, Finite-Element-Method

\section{Introduction}

The present study investigates the effect of using a modified drawing die geometry for cold drawn wires to influence the residual stresses of the wire. The aim is the reduction of tensile residual stresses near the surface.

Wire drawing is a method of bulk metal forming. The diameter is reduced by successive passes through a set of conical dies. Residual stresses are generated due to the inhomogeneous plastic deformation [1]. These stresses influence the mechanical behaviour of the wires and their durability and have an important effect on the functional properties of the wire for further forming operations or applications [2]. In general, residual stresses in the macro and micro range affect the strength properties. The superposition principle can lead to macro residual stresses and external load stresses exceeding the yield point of the material prematurely. However, in case of technical application this principle could be used to absorb a local treatment by elastic deformation because of reversal of the sign of the residual stress in comparison to the load stress [3]. Scholtes [4] stated that the vibration resistance caused by the development of residual compressive stresses could be improved by using shot peening or deep rolling. These additional processes influence the residual stresses after wire drawing. With regard to the optimization of the fatigue strength, residual compressive stresses are more suitable than tensile residual stresses due to reduced stress intensity. Llorca et al. [5] demonstrated that tensile residual stresses on the wire surface reduce the fatigue strength. In order to optimize the fatigue strength, the profile of the residual stresses should be customized to the load distribution since the external forces, torques and the residual stress profile of the wire get to a stress condition which determines the fatigue behaviour under vibration stress.

Tekkaya [6] described and compared how the residual stress was influenced by changing the percentage reduction of the wire diameter in one conical die. The final state of residual stresses is influenced by numerous parameters in the wire drawing process. The present study was intended to investigate the effect of modified drawing geometries of the die in the first section of drawing. Therefore the finite element method (FEM) was used. He et al. [2] investigated the influence of texture-based material models for simulated residual stresses after wire drawing. The computed distribution of the residual stresses over the cross section was evaluated using experimental specimens measured by X-ray

Corresponding author: markus.baumann@mb.tu-chemnitz.de 
diffraction. The measured and simulated residual stresses showed radial, tangential and axial residual compressive stresses in the wire core. When examining the cross section, it becomes apparent that the tangential and axial residual stresses are transformed into tensile stresses in the area from the wire core to the surface of the wire. The radial residual stresses were zero. Eutectoid steel $(0.07 \% \mathrm{C})$ was used as the investigated material. The level of the residual stresses can be influenced as described in [7]. The increase of the residual stresses was achieved by minimizing the drawing steps. Överstam [8] investigated the influence of production tolerances and die geometry on the residual stresses. In [9] and [10] the modification of the approach angle and form of the die was determined and compared to the resulting residual stresses.

The influence of changing the die geometry using extrusion was investigated in [11]. The authors describe the principle of severe plastic deformation (SPD) for the process of gradation extrusion. To achieve an ultrafine grain size of below one micrometer in the peripheral layer near the surface, repeated deformation of the material has to be realized while changing the forming direction. The design of the die includes different sections providing the specific forming conditions. An aluminium alloy was used in the test series [12].

\section{Materials and method}

The aim is to apply the experiences from gradation extrusion to wire drawing. Therefore the main results of gradation extrusion are presented first. Afterwards the methods of the FE simulations and the modified die geometries are described.

\subsection{Gradation extrusion}

Gradation extrusion (GE), a process which combines common impact extrusion with severe plastic deformation, is used to create property graded materials (Fig. 1). The processed material exhibits an increased strength in the near-surface layers and a relatively ductile core with lower strength [11].

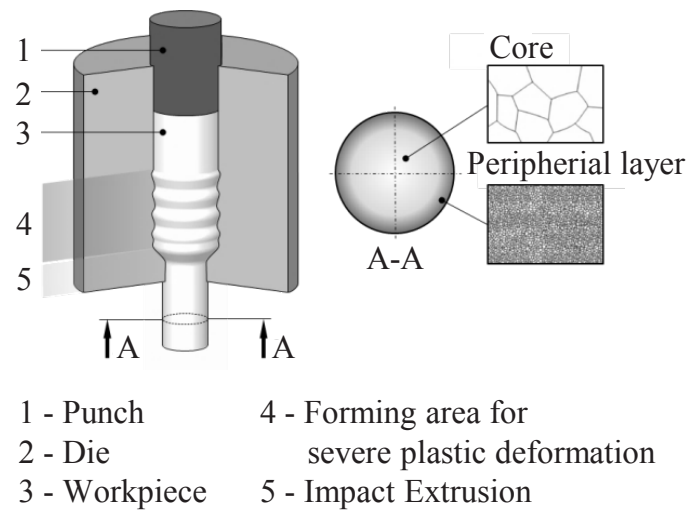

Fig. 1. Principle of gradation extrusion [12]
The aim of the GE process is to provide a severe plastic deformation process which has a high potential for applications in industrial manufacturing. It is intended to be integrated into process chains and to render the advantageous properties of the material accessible for various fields of applications. Therefore the process was designed to be material efficient, productive and applicable on standard machinery. Ideally, the forming process can combine both the microstructure modification and the generation of the geometry. In order to meet these aims, the basic approach consists in integrating severe plastic deformation into a common extrusion process and focusing the severe plastic deformation of the material to a local area with the highest load bearing requirements, which is generally the lateral area of a part. The name gradation extrusion refers to the major characteristic effect of the method: the generation of a graded microstructure. The processed workpiece possesses an ultrafine grained microstructure in the lateral area which gradually becomes coarser towards the middle or core of the workpiece where the grain size corresponds to the initial coarse or normal microstructure. The distribution of mechanical properties basically corresponds to the microstructure gradation [13].

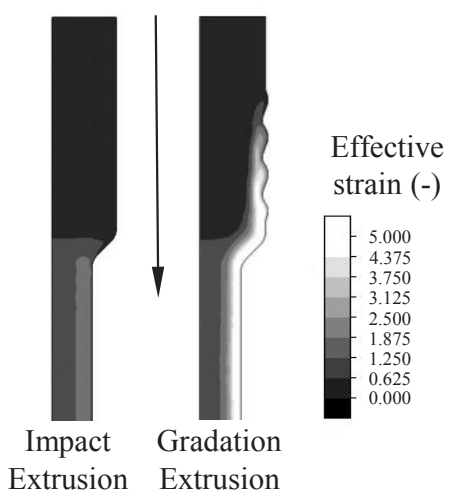

Fig. 2. Distribution of effective strain determined with FE simulation [14]

Fig. 2 shows the distribution of effective strain obtained from the FE simulation representing the steady state of material flow. A pronounced gradient of effective strain was found, ranging from the interior region to the surface. This gradient increases with every forming element in axial direction. The final cross-sectional reduction leads to an additional introduction of strain, resulting in a final gradient ranging approximately from 1.1 (interior) to 4.9 (surface). The process basically fulfils the requirements for severe plastic deformation processes (SPD processes), since it includes large effective strains, load path changes, high hydrostatic pressure and temperatures below recrystallization temperature. These results show that processing by gradation extrusion has a high potential for grain refinement by SPD, which leads to the assumption that distinct microstructural gradients may occur [15]. These previous studies demonstrate that special geometries influence the microstructure and the effective strain. The results should be transferred to the wire drawing process. 


\subsection{Finite element simulation}

The investigations described in this paper were conducted using the finite element program ABAQUS 6.14. The forming process was simulated with an explicit solver. To calculate the residual stresses of the wire after forming, the tool was removed and an implicit simulation of the wire followed. Using a defined node set over the cross section in the middle of the wire the axial, tangential and radial residual stresses were determined. Due to the axial symmetry of the wire drawing process, an axially symmetric two-dimensional FE model was set up (Fig. 3). The initial diameter of the wire was $12 \mathrm{~mm}$, and resulted after forming in a diameter of $10.8 \mathrm{~mm}$, which means a effective strain for the process of $\varphi=0.2107$. The conventional die has a reduction die angle of $12^{\circ}$. In the simulation the workpiece consists of 4200 elements with an element size of the mesh, which is $0.1 \times 0.5 \mathrm{~mm}^{2}$ in the peripheral region and $0.5 \times 0.5 \mathrm{~mm}^{2}$ in the core. The results of the FE simulations depend on this size of the mesh. Due to the expected high degree of deformation, the near surface layer of the wire was modelled using adaptive mesh refinement. In order to minimize calculation time, the die is defined as a rigid body in this studies. Further, elastic-plastic properties of the die require investigations such as mechanical characterization and experimental tests to validate the obtained results.

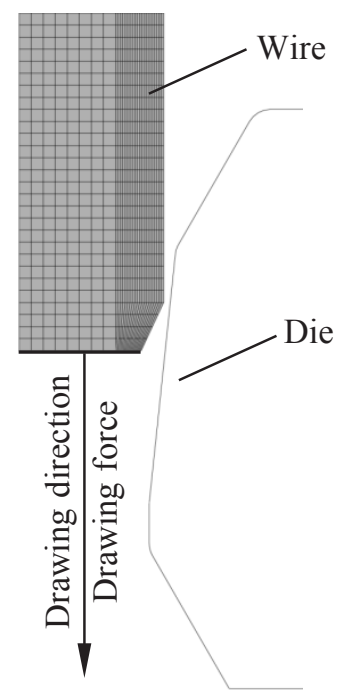

Fig. 3. Axially symmetric two-dimensional FE model for simulations of wire drawing

The austenitic steel X5CrNi18-10/1.4301 was defined for the simulations. The flow curve was established by using Hensel-Spittel approach (1).

$$
\begin{aligned}
& k_{f}=A \cdot e^{m_{1} \cdot \theta} \cdot \vartheta^{m_{\mathrm{g}}} \cdot \varphi^{m_{2}} \cdot e^{\frac{m_{\mathrm{q}}}{\varphi}+m_{7} \cdot \varphi} \cdot(1+\varphi)^{m_{\mathrm{s}} \cdot \theta} \cdot \dot{\varphi}^{m_{\mathrm{g}}+m_{\mathrm{g}} \cdot \theta} \text { (1) } \\
& \mathrm{A}=1188.736, \vartheta=20 ; \mathrm{m}_{1}=-0.00173 ; \mathrm{m}_{2}=0.29211 \text {; } \\
& \mathrm{m}_{3}=0.00456 ; \mathrm{m}_{4}=0.00021 ; \mathrm{m}_{5}-\mathrm{m}_{9}=0 ; \dot{\varphi}=0.0018 \text { [16] }
\end{aligned}
$$

Young's modulus, E, was set to $198.7 \mathrm{GPa}$ and Poisson's ratio, $v$, was set to 0.3 . The material for these investigations was assumed to be isotropic and elastic-plastic. The influences of the temperature and the strain rate were at first not considered in the modelling, despite the fact that they would influence the flow rate. Further studies are planned using special material models as described in [2] and elastic-plastic modelling of the die. The material models will consider the temperature behaviour, the microstructure, the strain rate and texture of the material. The investigation described in this present paper focused on the fundamental relationships between the residual stresses in the wire after drawing regarding the die geometry by using the defined mesh size of the wire and friction behaviour to the die. The friction between the wire and the die was modelled according to Coulomb's law with a friction coefficient of $\mu=0.03$ [2]. The drawing direction was determined by a boundary condition at the bottom of the wire (Fig. 3). In this context, certain displacement was defined at the 29 respective nodes. The drawing force is evaluated as a sum using these nodes. The penalty formulation was defined as a contact constraint to the die.

\subsection{Modified die geometry}

The developments of different die geometries are based on a conventional die for wire drawing with an reduction die angle of $12^{\circ}$. The wire diameter of each investigated die geometry is reduced from $12 \mathrm{~mm}$ to $10.8 \mathrm{~mm}$. The cylindrical part, also referred to as bearing length, is implemented with the same length of $1.64 \mathrm{~mm}$ at each geometry variant. Influences of different bearing geometries are investigated by Överstam [8]. To identify the correlation between the drawing die geometry and resulting residual stresses a single forming element was realized. Three different die geometries are investigated with two variants $(\mathrm{V})$ each. Fig. 4 presents a comparison between the convex, concave, step (each V1 and V2) and the conventional geometry.

The convex geometry V1 should cause deformation in the diameter of a conventionally formed die from $12 \mathrm{~mm}$ to $11.30 \mathrm{~mm}$ with a reduction die angle of $12^{\circ}$ and further severe deformation down to $10.8 \mathrm{~mm}$ by using a forming angle of the element of $60^{\circ}$. The size of the forming element is $0.25 \mathrm{~mm}$ (convex V1). V2 of this convex geometry exhibits a forming angle of $15^{\circ}$ of the element by the same size of $0.25 \mathrm{~mm}$, which implies an initial diameter of $11.63 \mathrm{~mm}$ before the forming element. The geometry concave V1 is implemented with the same size, angle of the forming element and end position as the forming element in geometry convex V1. Further forming to a diameter of $10.8 \mathrm{~mm}$ is done with a conventional reduction die angle of $12^{\circ}$. At the geometry concave V2, the forming element is positioned in front of the bearing length at the end of the conventional forming. The forming element size is $0.25 \mathrm{~mm}$ with a forming angle of $60^{\circ}$. 

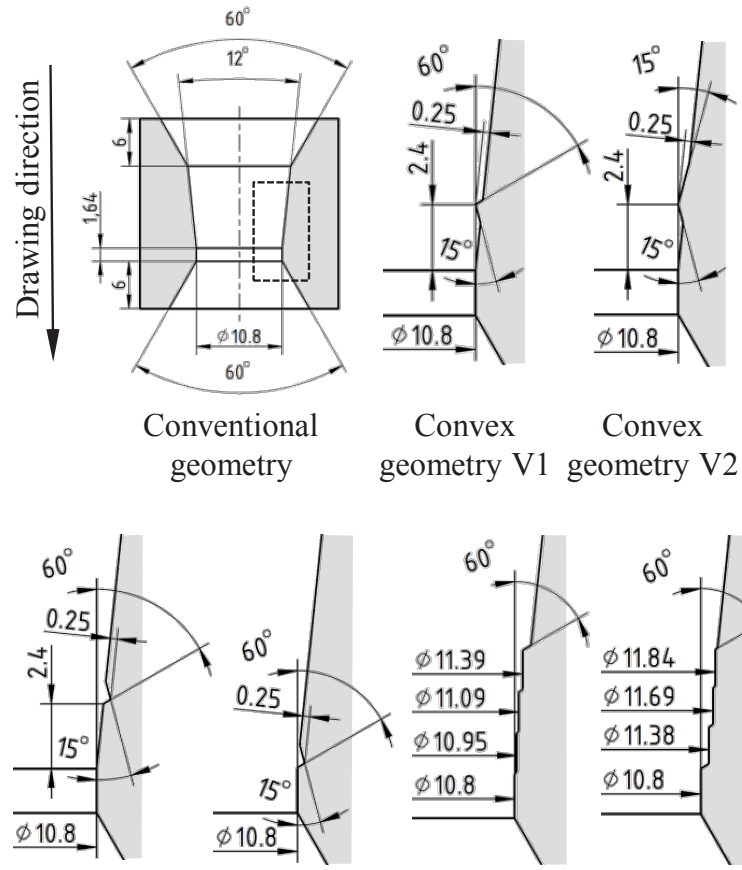

Concave Concave geometry V1

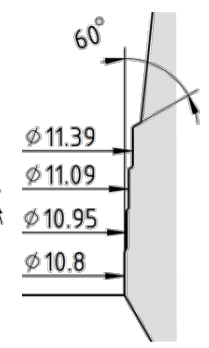

Steps

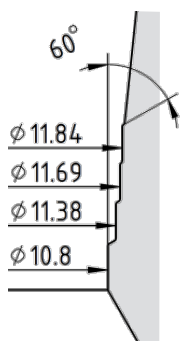

Steps

Fig. 4. Die geometries

The geometries step V1 and V2 comprise forming of the wire in four stages. The overall effective strain of the process $(\varphi=0.2107)$ is divided into step V1 with stage 1 entailing approx. half the overall effective strain $(\varphi=0.105)$, in stage 2 the effective strain value is $50 \%$ of the effective strain of stage $1(\varphi=0.0525)$, and stage 3 and 4 each comprise one half of the remaining effective strain $(\varphi=0.0266)$. In step V2 the stage geometry is inverted $(0.0266,0.0266,0.0525,0.105)$. The distance between each stage is $1.5 \mathrm{~mm}$.

$\mathrm{R}=0.1 \mathrm{~mm}$ applies to the active forming radii of each forming element of the different geometries and variants. The geometry for gradation extrusion presented by Bergmann [14] was not designed. Contrary as shown in Fig. 1, the contour of the forming element will not be filled in due to the tensile forces in wire drawing. The first geometrical element in the drawing direction causes an overall severe reduction of the wire diameter, or induces cracking of the wire.

\section{Results of FE simulation}

In order to identify the effect on residual stresses after drawing by the differently designed die geometries, the axial, radial and tangential stresses are evaluated. The following figures present the results. The values of the stresses are analysed at nodes in radial direction in the middle of the drawn wire. It has to be considered that the results of the residual stresses depend on the defined size of the mesh, the material model and further named boundary conditions.

Fig. 5 shows the residual stresses which occur when applying a conventional die geometry in wire drawing. The stress distribution of the axial and tangential directions exhibits compressive residual stresses in the core of the wire and transforms into tensile residual stresses on the surface. No radial residual stresses are detected on the surface of the wire. These residual stress distributions are typical for conventional wire drawing processes [1]. The axial and tangential tensile residual stresses on the surface of the wire are critical for further forming operations such as bending of the wire, as applied for producing torsion bars.

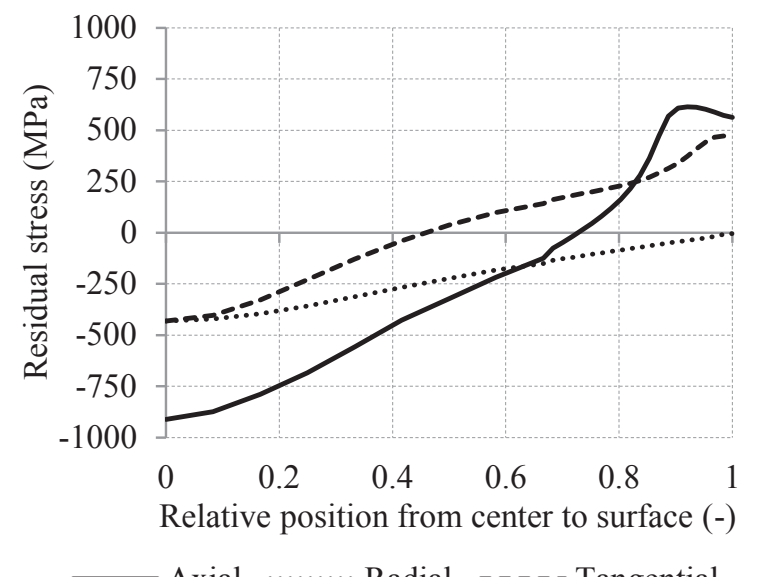

Fig. 5. Results of residual stress for conventional geometry

The die geometry for the first drawing step was changed (Fig. 4) in order to influence the residual stress distribution of the wire. Fig. 6 compares the results of the FE simulation for convex geometries. The radial stresses of the two geometrical variants show approximately the same values and flows. Large deviations regarding axial and tangential residual stress occur between convex V1 and V2.

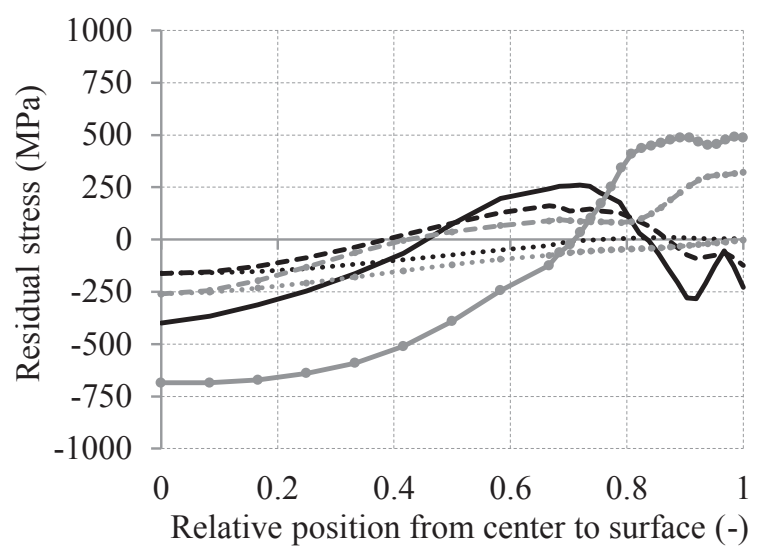

Convex geometry V1

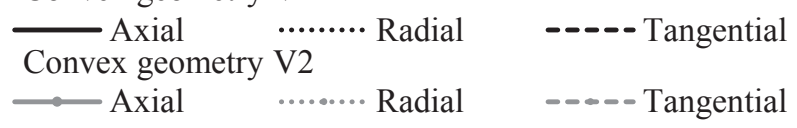

Fig. 6. Results of residual stress for convex geometry 
By using a convex element with an angle of $60^{\circ}$ and a size of $0.25 \mathrm{~mm}$ of the forming element as it is the case in convex V1, the residual stresses on the surface change to compressive axial and tangential residual stresses compared to forming with the conventional die geometry. The forming element has an influence on the overall stress distribution. The compressive stresses in the core are halved and zero crossing occurs much earlier compared to the stress flow of the residual stress of wire forming due to conventional die geometry. In convex V2, which implies an angle of $15^{\circ}$ of the forming element instead of $60^{\circ}$ in convex V1, the stresses in the core are reduced, but the tensile residual stresses at the surface are not minimized to zero or compressive stresses, compared to the conventional geometry shown in Fig. 5. The analysis of the material flow in the FE simulations showed that the material does not flow back in wire drawing after shaping the convex forming element. The convex geometry affects the forming of the wire in the same manner as a die with large reduction die angle.

Fig. 7 presents the results of the residual stress distribution by forming the wire by conventional geometry with an additional concave forming element. Concave V1, with a concave forming element in the position of the geometry convex V1 shows approximately the same stress distribution as in forming with a conventional die. Only a small forming effect was detected in the FE simulation analysis. Moving the forming element down to the end of the reduction zone of the conventional die geometry before the bearing starts, which is realized in the geometry concave V2, there is a large influence on the stress distribution of axial, radial and tangential residual stresses compared to results of conventional geometry. The compressive stresses in the core are halved compared to the conventional geometry and the zero crossing occurs much earlier. In addition, there is a severe decline from the tensile residual stresses to compressive residual stresses in the stress distribution to the surface of the wire, followed by an increase to zero and drop back to the range of compressive stresses.

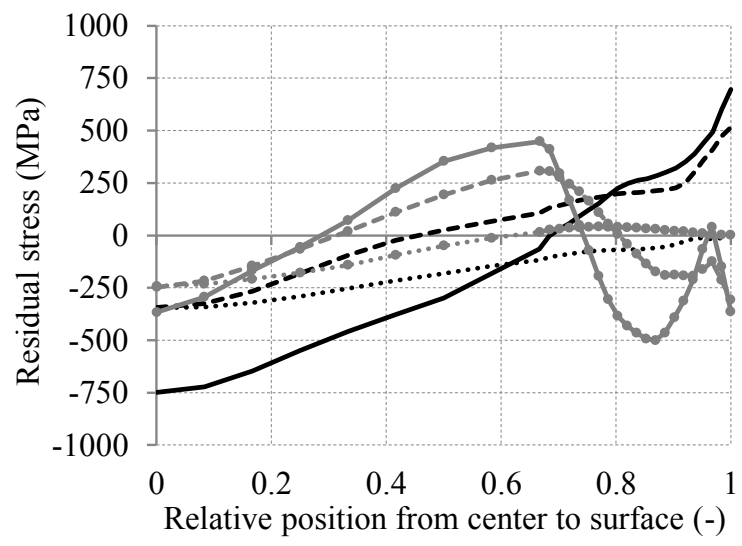

Concave geometry V1

$$
\begin{aligned}
& \text { Concave geometry V2 } \\
& \longrightarrow \text { Axial } \quad \text {........ Radial _-.-- Tangential }
\end{aligned}
$$

Fig. 7. Results of residual stress for concave geometry
The most significant influence on the residual stress distribution is achieved with the forming by step geometry. Fig. 8 demonstrates that over a large area of the cross section the stress distribution is in the range of compressive stresses.

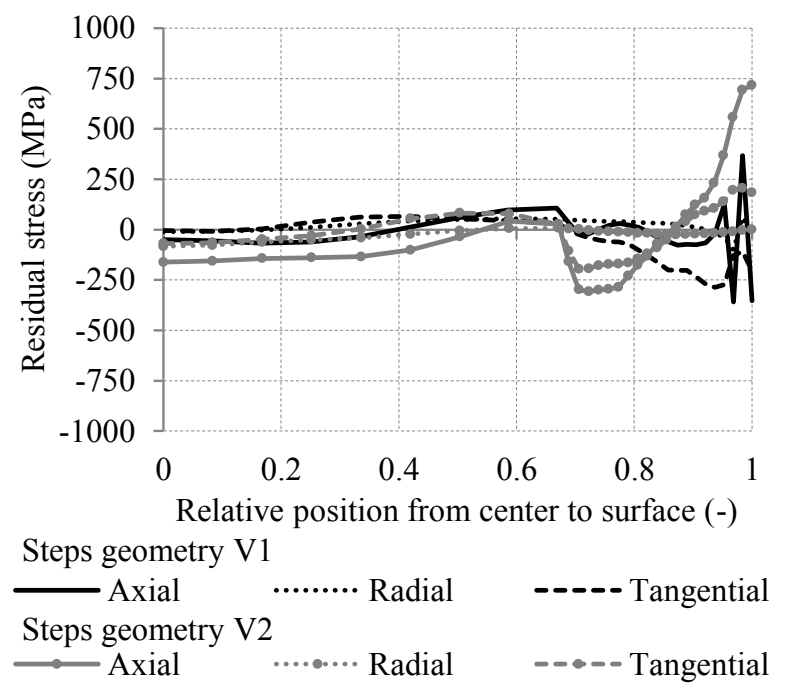

Fig. 8. Results of residual stress for steps geometry

Forming with the geometry step V2, which means a large strain rate occurs in the last stage, causes tensile residual stresses on the surface of the wire. When using the geometry step V1, which exhibits the largest strain rate in the first stage, compressive tangential stresses and varying axial stresses near the surface are implied. The differences occur due to multiple change of forming direction compared to the convex and concave geometries which is recognizable in the higher effective strain.

Because the axial and tangential tensile residual stresses at the near surface layer of the wire are critical for further forming operations, such as bending of the wire, these results of the distribution are compared in separate charts. Fig. 9 demonstrates the axial residual stresses of the different geometry variants compared up to $30 \%$ under the surface of the wire. In Fig. 10 the tangential residual stresses are compared dependent on the different geometry variants.

Considering the defined mesh size, the material model and further named determinations of the FE simulations, Fig. 9 and Fig. 10 demonstrate that the aim of the studies, to generate compressive axial and tangential residual stresses in the near surface layer, is achieved with the geometries convex V1, concave V2 and step V1. On the basis of these results the aim for further studies is to combine and investigate more geometry variants to deduce tendencies of the individual geometry parameters. The aim of the presented studies was to investigate whether geometrical changes on the forming surface of the die influence the residual stresses of the wire in one drawing step. 


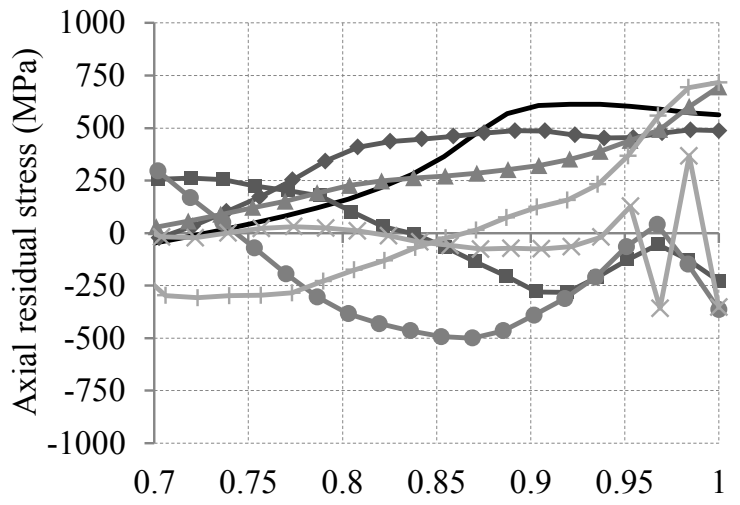

Relative position from center to surface (-)

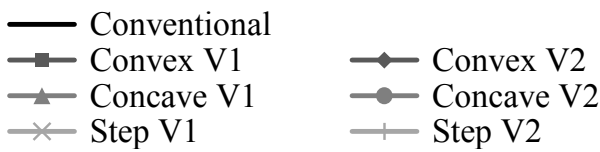

Fig. 9. Comparison of the axial residual stresses of the different die geometry variants

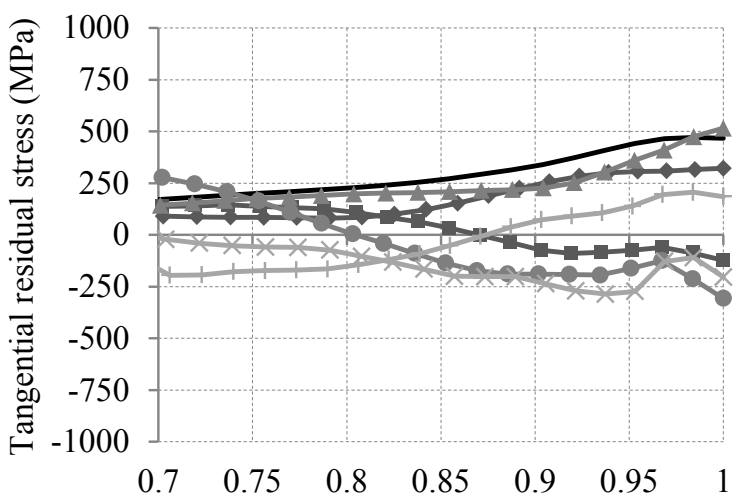

Relative position from center to surface (-)

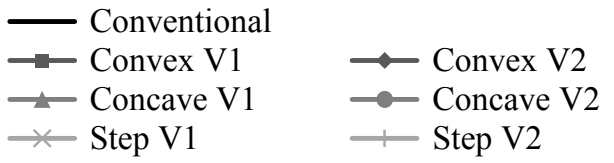

Fig. 10. Comparison of the tangential residual stresses of the different die geometry variants

To analyse the distribution of effective strain over the cross section and especially in the near surface layer the values are plotted by using the same node set as the residual stresses are evaluated. In Fig. 11 the effective strain curves, which are the results by forming the wire with the defined mesh size, are shown. The effective strain value in the core of all geometrical variants is $\varphi=0.21$. Nearly from the half of the wire diameter up to the surface the values of the effective strains are different. The maximum effective strain is reached by forming the wire with the step geometry. The absolute values of the effective strain depend on the relative position from the center to the surface. They are compared in Table 1.

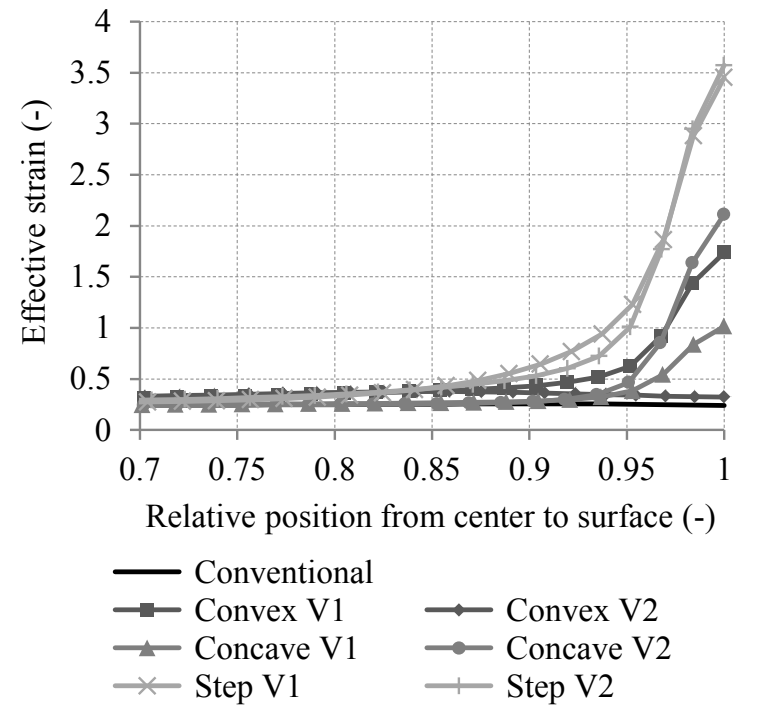

Fig. 11. Comparison of the effective strain at the near surface layer of the different geometry variants

Table 1. Effective strain dependent on relative position from the center to the surface

\begin{tabular}{|c|c|c|c|c|}
\hline \multirow[b]{2}{*}{$\begin{array}{c}\text { Geometrical } \\
\text { variant }\end{array}$} & \multicolumn{4}{|c|}{$\begin{array}{l}\text { Effective strain } \varphi \text { dependent on the } \\
\text { relative position from the center to } \\
\text { the surface (-) }\end{array}$} \\
\hline & 0.7 & 0.8 & 0.9 & 1 \\
\hline Conventional & 0.24 & 0.25 & 0.26 & 0.24 \\
\hline Convex V1 & 0.31 & 0.36 & 0.43 & 1.74 \\
\hline Convex V2 & 0.33 & 0.37 & 0.36 & 0.32 \\
\hline Concave V1 & 0.24 & 0.26 & 0.28 & 1.02 \\
\hline Concave V2 & 0.24 & 0.26 & 0.28 & 2.11 \\
\hline Step V1 & 0.27 & 0.34 & 0.64 & 3.45 \\
\hline Step V2 & 0.29 & 0.35 & 0.53 & 3.57 \\
\hline
\end{tabular}

Because of the multiple change of the forming direction the effective strain in the geometry variants step V1 and V2 is larger in the observed surface layers compared to the other geometrical variants. The variants with compressive residual stresses in the near surface layer (convex V1, concave V2 and step V1) show higher effective strain on the surface layer than the variants with tensile residual stresses at the surface layer. An exception is the geometry variant step V2. Further investigations are necessary to identify dependencies and tendencies of geometrical parameter influences.

Fig. 12 presents the results of average drawing forces values as function of the forming die geometry. Material modelling and the size of the defined mesh is important for the absolute values of the drawing force. However, it is possible to draw a relative comparison of the forces as function of the die geometry. The angle of the elements of the convex geometries (V1: $60^{\circ}, \mathrm{V} 2: 15^{\circ}$ ) has a 
significant impact on the resulting drawing force. Compared to the conventional die geometry, the geometry convex V1 showed an increase of approx. 70\% generated by the severe deformation of the forming element.

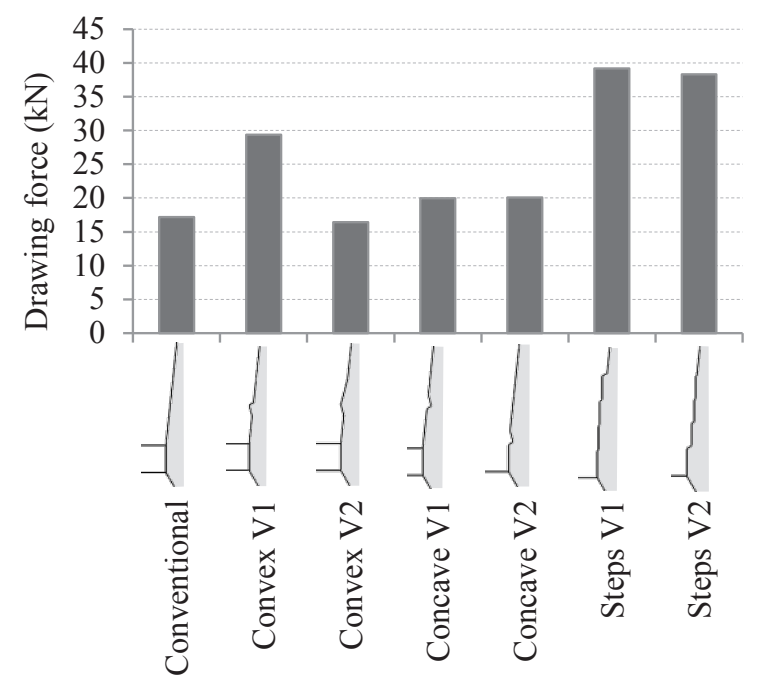

Fig. 12. Results average value of drawing force

The influence of the concave elements on the drawing force is not very significant. The forming elements of the step geometries have the greatest effect on the residual stress flow, but also the drawing force increases considerably because of the multiple change of forming direction due the step geometry. In addition the effective strain in the near surface layer is much higher.

\section{Conclusions and Outlook}

The aim of this investigations is to assess die geometry variants and to obtain geometries which reduce tensile residual stresses near the surface of the wire. The results of the FE simulation indicate that there are significant and different influences on the stress distribution of the residual stresses when using specific forming elements for wire drawing. It has to be considered that the results depend on the defined mesh size, the material model and the named boundary conditions. Regarding these conditions the pursued effect is achieved by using one convex forming element with a reduction angle of $60^{\circ}$ and an element size of $0.25 \mathrm{~mm}$. A concave forming geometry at the end of the forming could also lead to compressive residual stresses in the surface of the wire. The results of the axial and tangential residual stresses near the surface of the wire are compared in Fig. 9 and Fig. 10. The stepped forming geometries generate the lowest residual stress values in the core of the wire compared to the other geometries. However, more FE simulations with different die geometries and parameter variations are planned to deduce tendencies of the geometrical parameters on the resulting residual stresses of the wire. In these studies the limitations due to manufacturing constrictions such like radii or element sizes will be regarded.
In order to realize the planned further investigations, specific material models are required for the FE simulation. Isotropic hardening was assumed in these studies. The wire temperature, the forming ratio, the high strain rate because of drawing speed up to $100 \mathrm{~m} / \mathrm{min}$ and information about the texture of the microstructure have to be considered for wire drawing. In addition, experimental tests are essential to enable a validation of the simulations by a residual stress analysis by means of X-ray and neutron diffraction. Laboratory X-ray diffraction will be used to analyse the surface residual stresses and the residual stresses up to a depth of $500 \mu \mathrm{m}$ after different steps of electrochemical removal. Higher depths beyond $500 \mu \mathrm{m}$ up to the middle of the wire will be analysed by neutron diffraction. The analysis will yield the macro and micro residual stresses and their distributions over the wire diameter as well as information about the textures which are also important for the material modelling.

For the experimental work a test device shall be developed which will be implemented in a high speed testing machine. The die shall be interchangeable to use the test device for all drawing steps. Investigations of the behaviour of wear of the forming elements are part of advanced experimental studies. Using experimentally determined residual stresses by neutron diffraction and data from the experimental tests, the FE model can be validated by comparing numerical computations and experimental results [1]. As described, Neutron diffraction is necessary to determine the residual stresses in deeper layers of the wire.

The industrial wire drawing operations consist of more than one drawing step. FE simulations with four or five steps have to be computed and the residual stresses have to be evaluated.

In comparison to gradation extrusion, wire drawing is a different process due to the tensile forces in the forming direction. After forming by the convex forming element, the wire material does not flow back to the die. The planned experimental work will comprise the influence of the forming technology using specific forming elements in the drawing die.

The support provided by the priority program -SPP 2013-, which is financed by German Research Foundation, is gratefully acknowledged.

\section{References}

[1] J. M. Atienza, J. Ruiz-Hervias, M. L. MartinezPerez, F. J. Mompean, M. Garcia-Hernandez, M. Elices: Residual stresses in cold drawn pearlitic rods. Sript. Mat., 52, no. 12, pp. 1223-1228 (2005)

[2] S. He, A. Van Bael, S. Y. Li, P. Van Houtte, F. Mei, A. Sarban: Residual stress determination in cold drawn steel wire by FEM simulation and X-ray diffraction. Mat. Sci. and Eng., 346, no. 1-2, pp. 101-107 (2003)

[3] V. Hauck: Structural and Residual Stress Analysis by Nondestructive Methods, pp. 235 (1997), no. 1-2, pp. 101-107 (2003) 
[4] B. Scholtes: Eigenspannungen in mechanisch randschichtverformten Werkstoffzuständen: Ursachen, Ermittlung und Bewertung. DGM Informationsgesellschaft (1991)

[5] J. Llorca, V. Sánchez-Gálvez: Fatique limit and fatique life prediction in high strength cold drawn eutetoid steel wires. Fatique Fract. Eng. Mater. Struct, 12, no. 1, pp. 31-45 (1989)

[6] A. E. Tekkaya: Ermittlung von Eigenspannungen in der Kaltmassivumformung. Berichte aus der Umformtechnik der Universität Stuttgart (1986)

[7] D. J. Celentano, M. Palacios, E. L. Rojas, M. A. Cruchaga, A. A. Artigas, A. E. Monsalve: Simulation and experimental validation of multiplestep wire drawing processes. Finite Elem. Anal. Des., 45, no. 3, pp. 163-180 (2009)

[8] H. Överstam: The influence of bearing geometry on the residual stress state in cold drawn wire, analysed by the FEM. Journal of Materials Processing Technology, 171, no. 3, pp. 446-450 (2006)

[9] J. M. Atienza, M. Elices: Influence of residual stresses in the tensile test of cold drawn wires. Mat. and Struc., 36, no. 262, pp. 548-552 (2003)

[10] Ch. Siva Naga Teja, N. Guru Murty, P. Satish Reddy: FE Analysis of Wire Drawing Process with different die contours. Int. journal of scie. eng. and $a d v$. tech., 4, no. 2, pp. 134-143 (2016)

[11] R. Neugebauer, A. Sterzing, R. Selbmann, R. Zachäus, M. Bergmann: Gradation extrusion Severe plastic deformation with defined gradient. Mat.-wiss. u. Werkst., 43, no. 7, pp 582-588 (2012)

[12] M. Bergmann, A. Sterzing, D. Landgrebe: Influencing the gradient of material properties by gradation extrusion. Appl. Mech. and Mat., 794, pp. 166-173 (2015)

[13] R. Neugebauer, M. Bergmann: Local Severe Plastic Deformation by Modified Impact Extrusion Process. Steel Research International - Special Edition: 14th International Conference on Metal Forming, pp. 471-474 (2012)

[14] M. Bergmann: Verfahren zur Herstellung gradiert hochgradig plastisch umgeformter Werkstoffe. Verlag wissenschaftlicher Scripten (2013)

[15] D. Landgrebe, A. Sterzing, N. Schubert, M. Bergmann: Influence of die geometry on performance in gradation extrusion using numerical simulation and analytical calculation. CIRP Annals-Man. Tech., 65, pp. 269-272 (2016)

[16] M. Spittel, A. Spittel: Datasheet X5CrNi18-10 1.4301, Metal Forming Data of Ferrous Alloys deformation behaviour, 2C1, SpringerMat. (2009) 